\title{
The fragile X: a scanning electron microscope study
}

\author{
CHRISTINE J HARRISON*, ELSPETH M JACK+, TERENCE D ALLEN $\dagger$, \\ AND RODNEY HARRIS+ \\ From * the Department of Cell Biology and $\dagger$ the Department of Ultrastructure, \\ Paterson Laboratories, Christie Hospital and Holt Radium Institute, Manchester M20 9BX; and \\ $\ddagger$ the Department of Medical Genetics, St Mary’s Hospital, Manchester M13 OJH.
}

SUMmARY Scanning electron microscopy (SEM) has been used to study the fragile $\mathrm{X}$ chromosome. N The fragile site appears as an isochromatid gap in the majority of cases, confirming light microscope (LM) observations. SEM has allowed a more precise location of the fragile site to the $\mathrm{Xq} 27 \cdot 3 \mathrm{\infty}_{\infty}^{\mathrm{N}}$ region.

Light microscope (LM) studies have indicated that the fragile site on the $\mathrm{X}$ chromosome is most frequently observed as a non-staining gap of variable width, usually involving both chromatids. However, there is some uncertainty as to the exact position of this site. Sutherland ${ }^{12}$ defined it to be either in the Xq27 or Xq28 region. The enhanced resolution provided by scanning electron microscopy (SEM) has allowed both confirmation of the morphology and precise location of the fragile site by application of a technique used previously to karyotype human metaphases by SEM. ${ }^{3}$

\section{Materials and methods}

Heparinised peripheral blood, from a male patient known to have a fragile $\mathrm{X}$ chromosome, was cultured in TC 199 medium with Earle's Salts without serum supplementation and containing phytohaemagglutinin for 66 hours at $37^{\circ} \mathrm{C}$. A total of $0.01 \mu \mathrm{g} / \mathrm{ml}$ colcemid was added for $1 \frac{1}{2}$ hours. Cells were incubated in $75 \mathrm{mmol} / 1 \mathrm{KCl}$ for 5 minutes at room temperature followed by three washes in methanol-acetic acid fixative $(3: 1)$. Between the second and third washes cells were incubated for 1 hour at $-20^{\circ} \mathrm{C}$. Chromosome spreads were made by dropping suspension onto coverslips, followed by air-drying. Several coverslips were stained in $2 \%$ Giemsa in Gurr's buffer (pH 6.8) for 8 minutes at room temperature for $\mathrm{LM}$ analysis.

Two to three days later the remaining coverslips were $G$ banded using a method modified from the technique of Seabright ${ }^{4}$ as follows: coverslips were incubated in $0.025 \%$ trypsin in $0.85 \%$ saline for

Received for publication 21 December 1982.

Accepted for publication 24 January 1983.
20 to 40 seconds at room temperature. Some of these specimens were Giemsa stained and mounted $\stackrel{\complement}{\infty}$ onto slides for LM analysis. Unstained coverslips $\overrightarrow{-}$ for SEM were fixed in 3\% glutaraldehyde in $0.1 \%$ mol/l Sorensen's phosphate buffer $\left(\mathrm{pH} \mathrm{7.4)^{5 }}\right.$ for a minimum of 30 minutes. These coverslips were processed by an osmium tetroxide/thiocarbohydrazide (osmium-TCH) fixation, which allowed direct $\overline{0}$ visualisation of uncoated preparations. ${ }^{6}$ This method $\stackrel{2}{\circ}$ was modified to suit our chromosome preparations. ${ }^{3} \stackrel{\square}{\Omega}$

Specimens removed from glutaraldehyde were $\overrightarrow{\vec{P}}$ rinsed in Sorensen's buffer and then treated as 3 follows: (1) fixation in $1 \%$ osmium in the same buffer for 10 minutes; (2) three rinses in distilled water; (3) 5 minutes' incubation in a saturated solution of TCH in distilled water; (4) three furtherô rinses in distilled water; and (5) additional fixation for 10 minutes in $1 \%$ osmium in distilled water. Steps (2) to (5) were repeated. The specimens were o then dehydrated through a graded acetone series then critical point dried from liquid carbon dioxide 을 with $100 \%$ acetone as the transitional fluid. Cover- $>$ slips were examined in a Cambridge $\mathrm{S} 4-10$ stereoscan and an ISI SS 40 electron microscope.

\section{Results}

CHARACTERISATION OF THE NORMAL $X$ CHROMOSOME BY SEM

The $\mathrm{X}$ chromosome is easily identified from the $\mathrm{C} \stackrel{0}{\odot}$ group chromosomes in a normal human metaphase $\stackrel{?}{?}$ spread by its characteristic $\mathrm{G}$ banding pattern (fig 1). 0 A relationship between the $L M G$ bands and the ${ }^{\circ}$ quaternary coiling of human metaphase chrom- $\mathbb{D}$ osomes, as observed in the SEM, has previously been $\mathbb{\mathbb { Q }}$ demonstrated. ${ }^{3}$ The circumferential grooves between 280 


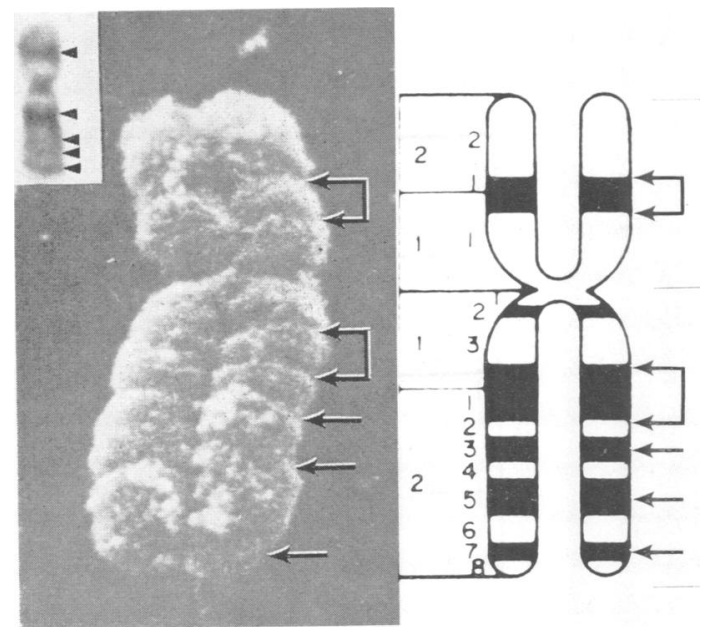

FIG 1 A normal $G$ banded chromosome viewed by SEM with a LM X chromosome (insot) prepared in parallel. The relationship between the $G$ positive $L M$ bands and the circumferential grooves, in accordance with the Paris nomenclature, is indicated by arrows. $(\times 7500$.

the gyres of the quaternary coils correspond to the $G$ positive bands, allowing easy recognition of the $\mathrm{X}$ chromosome in SEM preparations (fig 1).

SEM OF THE FRAGILE $X$

Characterisation of the fragile $X$

$\mathrm{G}$ banded human metaphase chromosomes, viewed

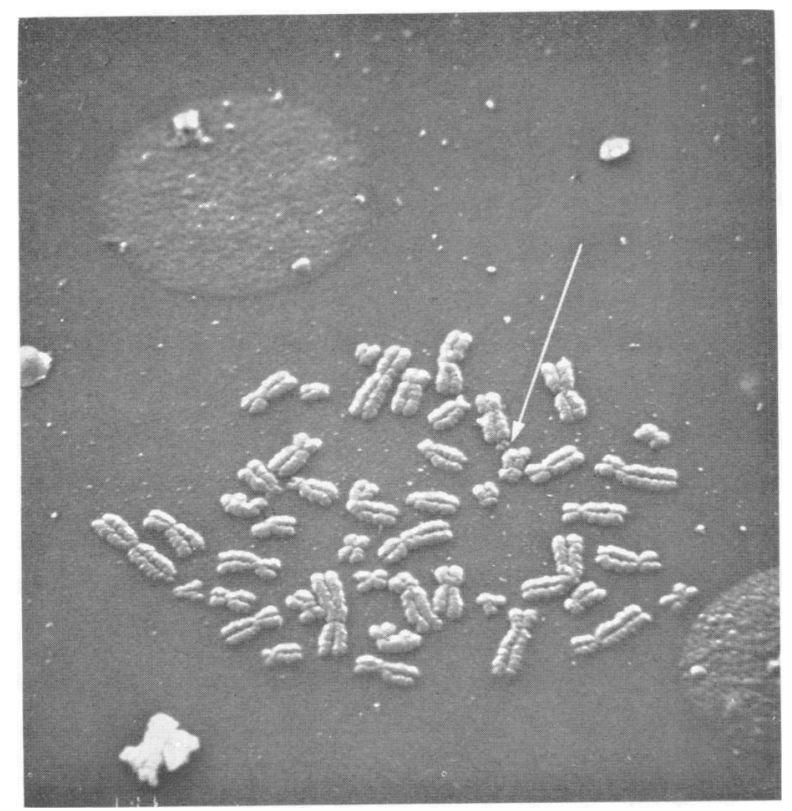

by SEM, showed a three dimensional cylindrical profile in which the fragile site of the $\mathrm{X}$ chromosome was prominent and readily detected (figs 2,3 ). In contrast, definition of the fragile site was reduced in $G$ banded metaphases in the LM (fig 3A, lower inset).

Two fragile $X$ chromosomes, from different $G$ banded metaphases, are shown in fig 3 (A, B). They illustrate the two types of fragile $X$ morphology observed when 100 metaphases were examined at random, both in the SEM and in the LM. The first chromosome (fig 3A) demonstrates almost complete breakage at the fragile site, producing an isochromatid gap with two fragments. The prominence of the isochromatid gap and the spherical nature of the fragments are demonstrated more clearly by SEM stereo photography (fig 4). This morphology was the type most commonly observed in SEM $(17 \%)$ and LM $(21 \%)$ preparations.

It was of interest to note that the end of the long arm of the $X$ chromosome, at the fragile site, had apparently contracted to form a rounded end, resembling the telomeres seen in normal chromosomes (fig 1). SEM observation showed that the fragments remained attached to the main part of the chromosome by individual fibres. The diameter of these fibres was approximately $25 \mathrm{~nm}$, consistent with the measurements of single fibres of chromatin.

A second type of fragile $\mathrm{X}$ morphology of reduced definition was less frequently observed, in which incomplete breakage occurred at the fragile site
FIG $2 A G$ banded metaphase spread. The three dimensional relief provided by SEM enhances the fragile site allowing easy identification of the fragile X chromosome. $(\times 1300$. $)$ 

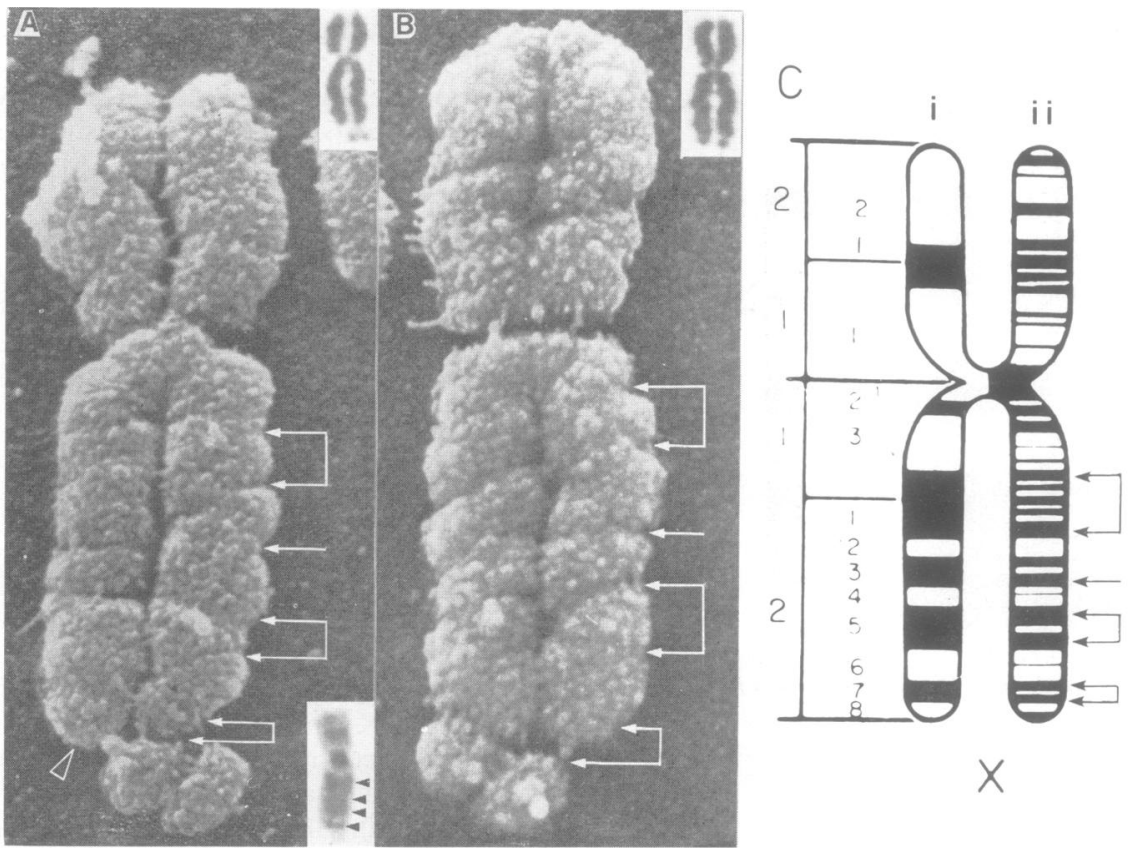

FIG 3 Two $G$ banded fragile $X$ chromosomes from different metaphases on the same coverslip viewed by SEM. The upper LM insets show non-banded fragile X chromosomes from the same person, showing the consistency of the gross morphology of the fragile sites when viewed by SEM and LM. The lower LM inset shows a Ganded fragile $X$ chromosome. The major $G$ positive bands are indicated by arrows as in fig $1 .(A)$ The fragile site appears as an isochromatid gap, both in the LM and SEM pictures, with the fragments almost completely separated. The deleted arm has formed a rounded end at the point of breakage (arrow head). The right hand chromatid has been displaced inwards in the Xq27 region. Note the interchromatid fibres between the fragments and the single fibres holding the fragments in close proximity to the deleted chromosome. $(\times 9500).(B)$ The fragile site appears as a chromatid gap on the right hand chromatid with the fragment completely detached. The fragment of the left hand chromatid is held in position by a series of fibres directly traversing the gap. $(\times 9500).(C)$ A diagrammatic representation of the $X$ chromosome ${ }^{8}$ showing the metaphase $G$ positive bands (chromatid $i$ ) subdivided into a series of sub-bands in the prometaphase chromosome (chromatid ii). The variation in intensity of staining of these bands is demonstrated by the thickness of the lines drawn. $(A, B, C)$ The relationship between the heavier stained, subdivided $G$ bands and the circumferential grooves is demonstrated by arrows within the Xq21, Xq23,Xq25, and Xq27 bands. From this correlation it is evident that the fragile site consistently arises at the lower Xq27 band (Xq27.3). This corresponds with LM observations (lower LM inset) in which the Xq27 band is complete above the fragile site.

(fig 3B and inset). A lower percentage was observed in the $\mathbf{L M}(1 \%$ in unbanded preparations, $0 \%$ in $\mathrm{G}$ banded preparations) than the SEM (7\%) preparations. The reduced resolving power of the LM may lead to uncertainty of diagnosis of this type of fragile site and therefore a lower percentage may be recorded. The high resolution of the SEM produced a clearer definition of these fragile sites with incomplete breakage. Fig 3B shows an example of this in the form of a chromatid gap at the fragile site with only one fragment completely detached. The other fragment was held in close association with the sister chromatid by a series of chromatin fibres. These appeared to have been 'pulled out' from the organised fibre arrangement of the chromatid.
Fibres in a similar pattern were observed $\underset{O}{2}$ between satellites and centromeres of acrocentric chromosomes.

A satellited D group chromosome is shown in fig 5 , which demonstrates the similarity in morphology between the fragments produced at the fragile site and the satellites themselves.

Interchromatid fibres were observed between the 0 two fragments (fig 3); since the two fragments were most frequently observed in close apposition to each? other the fibres may provide a function in maintaining this proximity.

Localisation of the fragile site It is now well established that the major LM G bands 


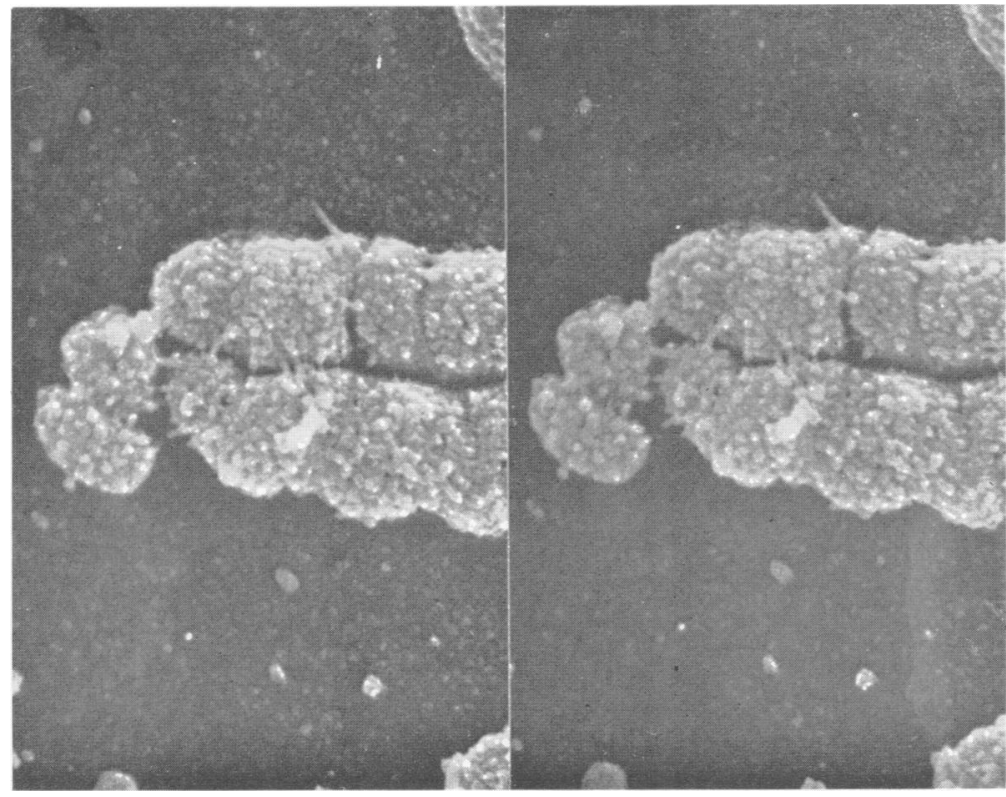

FIG 4 Stereo pairs of the $X$ fragile site. Stereo viewing demonstrates the isochromatid gap and the spherical nature of the fragments more clearly. ( $\times 9000$.)

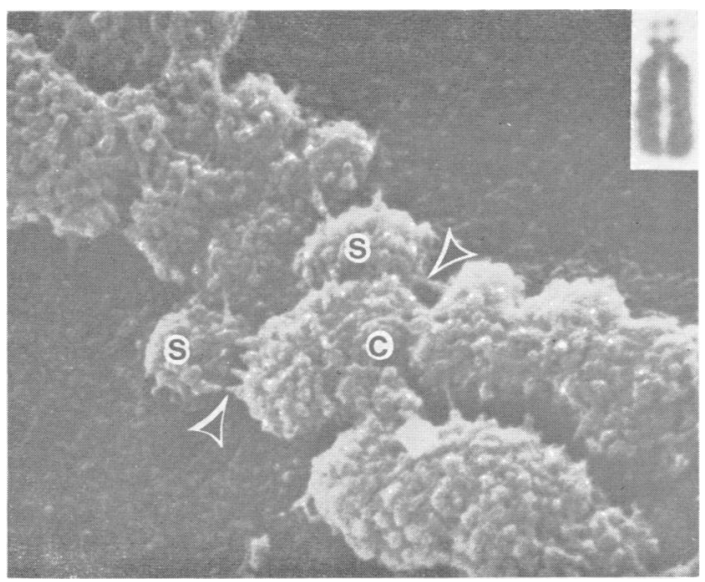

FIG $5 S E M$ and LM (inset) of the centromeric region $(C)$ and satellites $(S)$ of a $D$ group chromosome. Note the similarity in morphology between the satellites themselves and the fragments produced at the fragile site ( fig 3B). The fibres holding the satellites in position at the centromere (arrowheads) resemble those traversing the fragile site ( fig 3B). ( $\times 22500$.)

observed in metaphase chromosomes are made up of a series of smaller bands, as demonstrated in prometaphase chromosomes. ${ }^{7}$ We have previously shown that the large $G$ positive bands in the LM, for example those observed in the long arms of chromosomes 5,11 , and 12 , correspond to a series of closely associated circumferential grooves along both chromatids in the SEM. ${ }^{3}$ Therefore, the circumferential grooves observed in metaphase chromosomes in the SEM appear to correlate with the $G$ bands described for prometaphase chromosomes in the LM. This is demonstrated by observation of the $\mathrm{X}$ chromosome. Yunis ${ }^{8}$ showed, diagramatically, that the major $G$ positive bands of the metaphase $\mathrm{X}$ chromosome (as shown in chromatid (i), fig 3C, and the lower LM inset, fig 3A) were subdivided into a series of smaller bands in prometaphase chromosomes (as shown in chromatid (ii), fig 3C). These smaller bands showed variation in intensity of staining, the most prominent being represented by the heavier lines on chromatid (ii) (fig 3C). From examination of numerous fragile $X$ chromosomes, it was possible to demonstrate a relationship between these more prominent prometaphase bands (chromatid (ii), fig 3C) and the circumferential grooves in the SEM (fig 3A, B). This correlation was most evident in bands $\mathrm{q} 21, \mathrm{q} 25$, and $\mathrm{q} 27$.

The number of circumferential grooves in the long arms of these chromosomes was found to be the same but a shift in the position of these grooves, both between sister chromatids and between chromosomes, was observed (fig 3A, B). This may reflect the way in which the chromosomes made initial attachment to the surface of the coverslip during preparation, possibly leading to displacement 
of the chromatids, as demonstrated in the Xq27 region of the chromosome in fig $3 \mathrm{~A}$. The ability to resolve this type of variation in the SEM may help to explain the shifts in relative positions of the $G$ bands sometimes observed in the LM. Examination of numerous metaphases in the SEM revealed that the fragile site consistently occurred at the lower Xq27 sub-band, with no circumferential groove evident on the fragment itself. This was in agreement with LM observations which demonstrate the Xq27 band to remain complete above the fragile site, with no $G$ positive band present on the fragment (fig 3A, lower inset). Therefore, using SEM in parallel with LM findings, it has been possible to pinpoint the fragile site of the $X$ chromosome directly to the $\mathrm{Xq} 27 \cdot 3$ region.

\section{Discussion}

It has previously been reported that individual human metaphase chromosomes show an inherent specificity of quaternary coiling, which relates to the LM G banding pattern. ${ }^{3}$ By application of the same criteria, $\mathrm{X}$ chromosomes were identified by SEM. Examination of numerous normal and fragile $X$ chromosomes showed that the fragile site occupied a constant position. From the correlation of metaphase quaternary structure by SEM and the LM prometaphase and metaphase $G$ banding pattern, this site was found to be within the lower region of the $\mathrm{G}$ positive $\mathrm{Xq} 27$ band. This is referred to as the q27.3 band by Yunis et al $l^{7}$ and Yunis. ${ }^{8}$

The increased resolving power of the SEM has allowed the morphology of the fragile $X$ to be studied in greater detail. The three dimensional image, with the increased depth of field provided by SEM stereo photography, confirmed the fragile site as an isochromatid gap with spherical acentric fragments. The resemblance of these fragments to the satellites of D and G group chromosomes was clearly demonstrated by SEM.

At those fragile sites with incomplete breakage, or a single chromatid gap, the fragments were held in close proximity to the chromosome arms by a series of chromatin fibres. Groups of chromatin fibres cannot be resolved by $\mathbf{L M}$ and therefore difficulties may arise in identification of this type of fragile $\mathrm{X}$ from the normal $\mathrm{X}$ chromosome, resulting in an artificially reduced frequency. Since this fragile $X$ morphology was easily recognised by SEM a more accurate frequency of fragile $X$ may be obtained by the use of SEM in certain cases.

In those $\mathrm{X}$ chromosomes with almost complete breakage at the fragile site, single strands of chromatin retained the fragments adjacent to the 'deleted' chromatids. Interchromatid fibres held the fragments in close association with each other. $\stackrel{\mathbb{P}}{?}$ Therefore, the presence of chromatin fibres appears $\overrightarrow{\vec{F}}$ to influence the eventual morphology of the fragile $\mathrm{X}$ chromosome.

The mechanisms involved in formation of fragile $\frac{\overline{\bar{F}}}{\overline{0}}$ sites remain unknown. Sutherland ${ }^{1}$ and $\operatorname{Dakar}^{9} \frac{\bar{\sigma}}{\widetilde{\alpha}}$ made several suggestions. In view of our SEM $\cong$ observations, their most convincing proposal wasi that a failure of the complex folding of the chromatin $\vec{\circ}$ fibres occurred at the fragile site. The processesinvolved in the final stages of chromatin condensa- $\vec{\omega}$ tion into metaphase chromosomes are largely unknown, although several proposals have been⿸厃㔾 made.

Some of our earlier studies on chromosomal ${ }_{+}^{\circ}$ structural organisation $^{10}$ support the radial loopi model of chromosome construction, as proposed by ${ }^{\infty}$ Marsden and Laemmli. ${ }^{11}$ In this present study we음 demonstrate a loss of the organised structuralmorphology of the $\mathrm{X}$ chromosome in the region of $\square$ the fragile site, in which the fibres appear to haveo

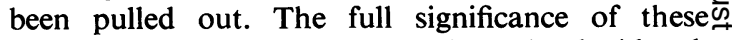
observed structural changes remains to be elucidated. $\overrightarrow{0}$

Further studies on other known fragile sites, $\underset{\omega}{\infty}$ carried out over the range of culture conditions, are needed in an attempt to understand further theo mechanisms of fragile site formation.

We thank Mrs E Grace and Mrs R Bauld, Cyto-市 genetics Laboratory, Royal Hospital for Sick Children, Edinburgh, for providing us with theo specimens and for initial identification of the fragile $\mathrm{X}$ chromosomes, and Dr M Hales of International Scientific Instruments for taking the high resolution micrographs. This work was supported by NWRHA project grant 418, the Cancer Research Campaign, $;-$ and the Leukaemia Research Fund.

\section{References}

1 Sutherland GR. Heritable fragile sites on human chromosomes. I. Factors affecting expression in lymphocyte culture. Am J Hum Genet 1979;31:125-35.

2 Sutherland GR. Heritable fragle sites on human chromosomes. II. Distribution, phenotypic effects and cyto-O genetics. Am J Hum Genet 1979;31:136-48.

3 Harrison CJ, Britch M, Allen TD, Harris R. ScanningO electron microscopy of the G-banded human karyotype. Exp Cell Res 1981;134:141-53.

4 Seabright M. A rapid banding technique for human chromosomes. Lancet 1971 ;ii:971-2.

5 Drury RAB, Wallington EA. Carleton's histologica/ technique. Oxford: Oxford University Press, 1980:495.

6 Ip W, Fischman DA. High resolution scanning electron $\square$ microscopy of isolated in situ cytoskeletal elements.우 J Cell Biol $1979 ; 83: 249-53$.

7 Yunis JJ, Sawyer JR, Ball DW. The characterisation of high resolution G-banded chromosomes of man. Chrom-을 osoma 1978;67:293-307. 
8 Yunis JJ. High resolution of human chromosomes. Science 1976;191:1268-70.

9 Dakar MG. X-linked mental retardation and the fragile X. Clin Cytogenet Bull 1982;1:3-14.

10 Harrison CJ, Allen TD, Britch M, Harris R. High resolution scanning electron microscopy of human metaphase chromosomes. J Cell Sci 1982;56:409-22.

11 Marsden MPF, Laemmli UK. Metaphase chromosome structure: evidence for a radial loop model. Cell 1979;17: 849-58.

Correspondence and requests for reprints to $\mathrm{Dr}$ C J Harrison, Department of Cell Biology, Paterson Laboratories, Christie Hospital and Holt Radium Institute, Manchester M20 9BX. 